\title{
Control de autoridades de los nombres propios \\ Los encabezamientos de materia en el Repositorio Institucional Nínive
}

\section{José Luis Montero O’ Farrill \\ Universidad de Moa Dr. Antonio Núñez Jiménez. Facultad de Ciencias Económicas. Centro de Estudios Pedagógicos. Moa, Cuba | jmontero@ismm.edu.cu / https://orcid.org/oooo-0o03-3421-4181}

\author{
Maylén Beatriz Pérez Pérez \\ Universidad de Moa Dr. Antonio Núñez Jiménez. Facultad de Ciencias Económicas. Departamento de Ciencias de la Informa- \\ ción, Cuba I mperezp@fh.ismm.edu.cu
}

\begin{abstract}
Niurka de la Vara Garrido
Universidad de Moa Dr. Antonio Núñez Jiménez. Facultad de Ciencias Económicas. Departamento de Ciencias de la Información, Cuba | nvara@ismm.edu.cu / https://orcid.org/oooo-00o2-9653-7616
\end{abstract}

\section{Flor María Reyes Hernández}

Universidad de Moa Dr. Antonio Núñez jiménez. Facultad de Ciencias Económicas. Departamento de Ciencias de la Información. Moa, Holguín, Cuba | freyes@ismm.edu.cu / https://orcid.org/oooo-0oo2-8064-7204

\section{Resumen}

La amplia cantidad de nombres propios utilizados en el proceso de indización, como términos para representar a los documentos, sumados a los casos de sinonimia, homonimia y variantes de la forma del nombre en el Repositorio Institucional Nínive de la Universidad de Moa Dr. Antonio Núñez Jiménez, hace necesario realizar el control de estos puntos de acceso para asegurar la coherencia del catálogo; $y$, así, facilitar el acceso a la colección documental. En esta investigación se elabora un catálogo que proporciona los puntos de acceso autorizados referente a descriptores: geográficos, aplicados a las ciencias informáticas y los nombres de las plantas industriales. Para ello se utilizaron los procedimientos tradicionales que se describen en la literatura científica para la creación y gestión de las autoridades: las Reglas de Catalogación Angloamericanas. $2^{\text {a }}$ edición, revisión de 2002 y actualización de 2003 [AACR2], las Directrices para Registros de Autoridad y Referencias [GARR], $2^{\text {a }}$ edición y las Directrices para los Registros de Autoridades de Materia y sus Referencias [GSARE]; así como obras de referencia léxicas. Se establecieron 25 lineamientos y se normalizaron 389 nombres propios. La investigación permitió elaborar un catálogo de autoridades que favorece la recuperación de la información en el Repositorio Institucional Nínive.

\section{Palabras clave}

Proceso de indización Control de autoridades Catálogo de autoridades Nombres propios Repositorio institucional 
Authorities's control of proper names: The subject headlines in the Repositorio Institucional de Nínive

\begin{abstract}
Keywords

Process of indexing Authorities's control Authorities's catalog Proper Names Institutional repository

The large number of proper names used in the indexing process as terms to represent the documents, added to the cases of synonymy, homonymy and variants of the name form in the Repositorio Institucional de Nínive of the Universidad de Moa Dr. Antonio Núñez Jiménez, makes it necessary to control these access points to ensure the coherence of the catalog; and to facilitate access to the documentary collection. In this research, a catalog that provides the authorized access points regarding geographical descriptors is elaborated, applied to computer science and the names of industrial plants. For this, the traditional procedures described in the scientific literature for the creation and management of authorities were used: the Anglo-American Cataloging Rules. 2nd edition, 2002 revision and 2003 update [AACR2], Guidelines for Registries of Authority and References [GARR], 2nd edition and Guidelines for Registries of Subject Authorities and their References [GSARE]; as well as lexical reference works. 25 guidelines were established and 389 proper names were standardized. The investigation made it possible to develop a catalog of authorities that favors the retrieval of information in the Repositorio Institucional de Nínive.
\end{abstract}

Artículo recibido: 22-05-2020. Aceptado: 23-10-2020

\section{Introducción. Contexto y origen de la investigación}

En la actualidad, el vertiginoso desarrollo de las tecnologías de la información y las comunicaciones y el creciente volumen de la información generada en la actividad científico informativa, ha propiciado que los Repositorios Institucionales (RI) tengan gran importancia para socializar y hacer visible la producción científica de los investigadores (Duperet Cabrera et al., 2015). En este contexto, el control de autoridades que determina la forma y estructura que tendrán los puntos de acceso con la uniformidad con que deben aparecer siempre, es una de las piezas clave a disposición de sus administradores para mejorar la calidad de los contenidos y posibilitar la interoperabilidad (Núñez Amaro, 2006).

El control de autoridades es un proceso que unifica mediante la utilización de una forma normalizada, los puntos de acceso de los catálogos automatizados y muestra las relaciones entre ellos. Normaliza los nombres de personas, entidades, títulos uniformes o materias, que pueden constituir el punto de acceso principal o los secundarios de este tipo de catálogo. Facilitando la identificación y recuperación de los documentos almacenados, evitando las confusiones a que se pueden prestar los homónimos, sinónimos o la variedad de nombres con los que puede ser denominado una persona, entidad, obra, tema o concepto (Martínez Tamayo et al., 1997).

El control de autoridades en los repositorios institucionales no es tarea fácil para los coordinadores de los mismos. La poca ayuda que han aportado los programas informáticos en sus primeras versiones y la propia esencia del autoarchivo, que plantea un sistema utilizado y manejado por miles de usuarios, hace que la normalización de temas o materias en un archivo digital abierto se haya considerado, por lo menos hasta ahora, misión difícil (Barrionuevo Almuzara; Alvite Díez y Rodríguez Bravo, 2011). 
Esta investigación tiene su esencia en el contexto del Repositorio Institucional Nínive, de la Universidad de Moa Dr. Antonio Núñez Jiménez de la República de Cuba, basado en la plataforma de software libre DSpace que se concibe como un servicio digital para favorecer la recogida, conservación y distribución de los recursos digitales relacionados con el aprendizaje, la enseñanza y la investigación; elaborados por sus miembros, bajo una política de acceso abierto.

En el caso de la documentación almacenada en el Repositorio Institucional Nínive de la Universidad de Moa Dr. Antonio Núñez Jiménez, no se desarrolló un proceso de normalización en este sentido, generando un índice de materias muy amplio y diverso en el cual existe una gran variedad de nombres propios lo que dificulta la consistencia en los metadatos de materia y la búsqueda y recuperación de la información.

Una búsqueda simple permite observar el alto nivel de inconsistencia presente en los índices de materia (Gráfico 1):

"El uso indistinto de nombres propios por parte de los autores de los documentos y de los indizadores al utilizar estos términos como encabezamientos de materia, en el proceso de indización.

» La falta de lineamientos y políticas precisas para realizar la indización de los documentos utilizando este tipo de términos.

» La inexistencia de un catálogo de autoridades de nombres propios utilizados como términos de materias para aplicar al Repositorio Institucional Nínive.

Gráfico 1. Existencia de múltiples variantes para designar un mismo término

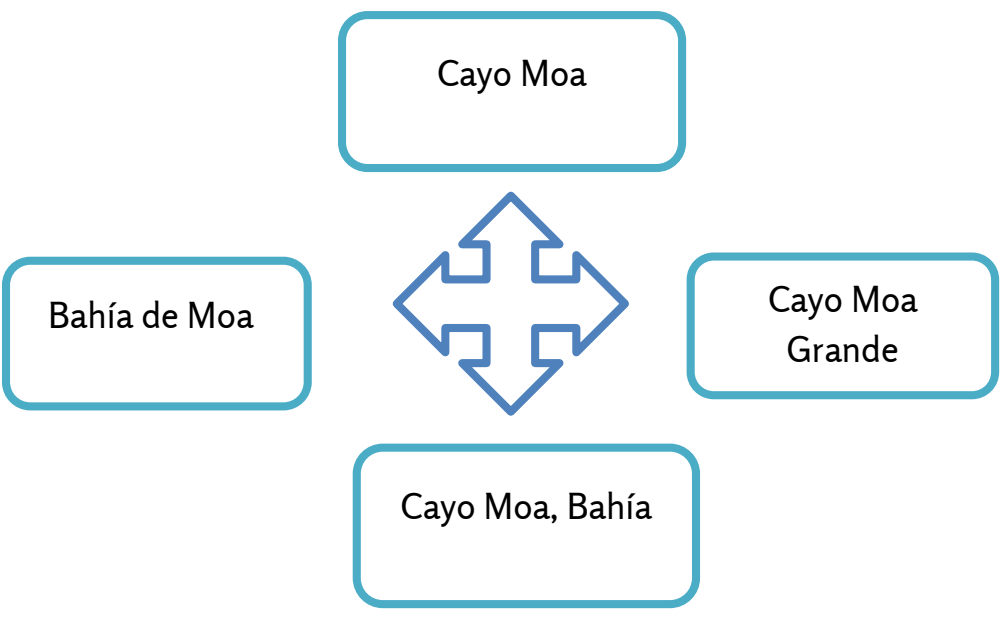

Fuente: Elaboración propia

Para solucionar este problema y favorecer la búsqueda y recuperación de la información en el Repositorio Institucional Nínive de la Universidad de Moa Dr. Antonio Núñez Jiménez se decidió establecer un control de autoridades de los nombres propios utilizados como encabezamientos de materia. 
El objetivo de este trabajo es elaborar el control de autoridades de los nombres propios utilizados como términos de materia para el procesamiento y la representación de la información en el Repositorio Institucional Nínive de la Universidad de Moa Dr. Antonio Núñez Jiménez; favoreciendo su acceso y recuperación.

\section{Metodología. Etapas de trabajo implementadas para elaborar el catálogo de autoridades}

Se utilizó el materialismo dialéctico e histórico como método general de investigación para analizar los fenómenos históricos y sociales que dieron lugar al lenguaje documental. Se explicaron los conceptos, métodos y herramientas que posibilitaron el desarrollo del vocabulario a partir del avance tecnológico facilitando el proceso de búsqueda debido al cúmulo de recursos informativos. Se utilizaron métodos teóricos y empíricos.

Teniendo en cuenta los procedimientos tradicionales que se describen en la literatura científica para la creación y gestión de las autoridades (Mesa León et al., 2007; Pugni, 2011; Firpo, 2014; Biblioteca Nacional de España, 2013; Maldonado Martínez y Rodríguez Yunta, 2004; Martínez Saldaña, 2006; García Rodríguez, 2016; Núñez Amaro, 2007), este proceso se efectuó siguiendo varios pasos:

1. Definición de los puntos de acceso.

2. Búsqueda y selección de las fuentes bibliográficas y obras de referencia a utilizar para la apoyar la investigación.

3. Selección de los expertos a revisar la terminología a normalizar.

4. Selección de las herramientas bibliotecológicas a emplear.

5. Establecer lineamientos para normalizar los términos.

6. Selección de la estructura del control de autoridades.

7. Presentación del Catálogo de Autoridad.

\section{Definición de los puntos de acceso a normalizar}

Uno de los primeros pasos que se definen en la literatura científica tomando en cuenta a Díaz Rodríguez (2012), es la definición de los puntos de acceso que serán sometidos al proceso de normalización para posteriormente descubrir todas las variantes posibles con las que puede nombrarse.

Dentro del proceso de control, la selección del nombre propio se basa en la condición de que solamente debe constituirse en autoridad una sola forma del nombre, independientemente de la cantidad de posibilidades bajo las cuales dicho nombre se conozca. La tarea más compleja es la de comprobar si un término que aparece en más de una variante se trata en verdad de la misma autoridad o no.

Para los efectos de esta investigación se decidió seleccionar en una primera etapa de trabajo aquellos nombres propios que son muy utilizados en las especialidades de la geología, minería, metalurgia e informática básicamente y que los usuarios en las solicitudes de información los utilizan con mucha frecuencia.

Para recolectar estos términos, se procedió a realizar búsquedas en el índice de materia de todas las comunidades presentes en el repositorio, identificando un estimado de 389 nombres propios. A partir de ello se estableció una división de estos nombres quedando de la siguiente manera (Gráfico 2): 
Gráfico 2. Clasificación de los descriptores a normalizar

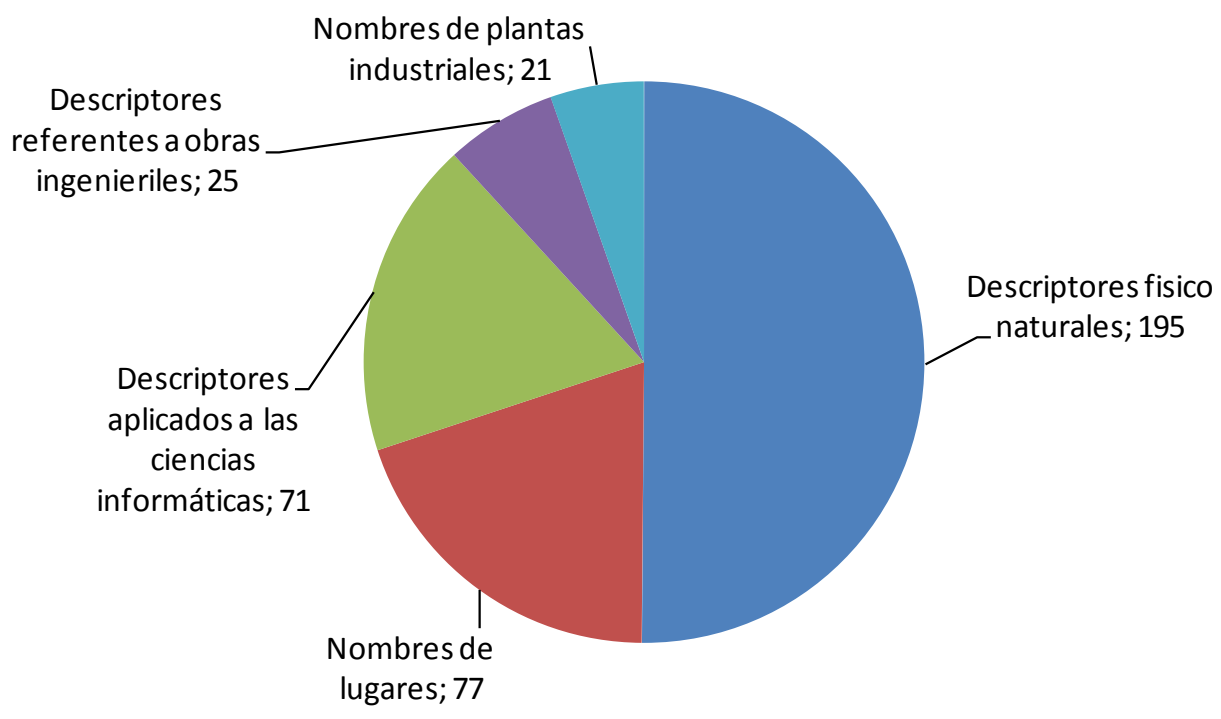

"Nombres de plantas industriales

" Nombres de descriptores geográficos:

» Nombres de descriptores físico naturales (ríos, cuencas, lagos, bahías, arroyos, yacimientos, canteras, formaciones geológicas, minas, sectores, valles).

» Nombres de descriptores referentes a obras ingenieriles (presas, túneles, carreteras, parques eólicos, pozos).

"Nombres de repartos, poblados, municipios, provincias, y países.

»Nombres de descriptores aplicados a las Ciencias Informáticas (nombre de software, base de datos, aplicaciones informáticas y de lenguajes de programación).

\section{Búsqueda y selección de las fuentes bibliográficas y obras de referencia a utilizar para la apoyar la investigación}

Como referente para esta investigación se encontró escasa bibliografía, ya que la mayoría de estas investigaciones están dirigidas generalmente con el control de autoridades referentes a los nombres de los autores de los documentos, a los nombres de entidades y a los nombres geográficos de lugares, por lo que se decidió en este trabajo utilizar como guía: el "Manual de indización para Archivos del Archivo Nacional de la República de Cuba" (Mesa León et al., 2007) y "Proceso de creación de una lista de autoridades de nombres geográficos de Argentina" (Pugni, 2011) que respalda la misma idea llevada a cabo en esta investigación; realizándole las adaptaciones necesarias al contexto de la institución.

Entre las herramientas léxicas utilizadas para la revisión de los nombres se mencionan: Nombres geográficos de Cuba. Editorial Pueblo y Educación (Acevedo Rodríguez y León Herrero, 2013); Nomenclátor de Nombres Geográficos Normalizados de la provincia de Holguín (Comisión Provincial de Nombres Geográficos, 2013); Diccionario de nombres geográficos de la provincia de Holguin (Bacallao González; Garzón Montes de Oca y Bacallao González, 2011) y EcuRed. Enciclopedia Cubana http://www.ecured.cu/

\section{Selección de los expertos a revisar la terminología a normalizar.}

A través de la aplicación de técnicas de recopilación de la información, en este caso la entrevista, se utilizaron como fuentes de información a especialistas de las carreras de minería, geología, metalurgia e informática para revisar los nombres propios 
utilizados, así como conocer de la existencia de otra terminología equivalente para nombrar a estos términos. En el caso de los nombres de descriptores físico naturales y descriptores geográficos referentes a obras ingenieriles, muy utilizados por la comunidad de usuarios de geología y minas, se necesitó conocer su lugar de ubicación de forma precisa.

Tabla 1. Especialistas que revisaron la terminología para el control de autoridades

\begin{tabular}{llc}
\hline Grado científico & Especialidad & Años de experiencia \\
\hline Doctor en Ciencias & Minería & 40 \\
Doctor en Ciencias & Minería & 35 \\
Doctor en Ciencias & Metalurgia y materiales & 35 \\
Doctor en Ciencias & Metalurgia & 13 \\
Doctor en Ciencias & Geología & 38 \\
Máster en Ciencias & Geología & 11 \\
Doctor en Ciencias & Informática & 36 \\
\hline
\end{tabular}

\section{Selección de las herramientas bibliotecológicas a emplear}

Para establecer los lineamientos a seguir en el proceso de indización con el uso de este tipo de términos se utilizaron: las Directrices para registros de autoridad y referencias (GARR), $2^{\mathrm{a}}$ edición revisada por el Grupo de Trabajo de la IFLA (International Federation of Library Associations), traducción de Justo García Melero; Directrices para los registros de autoridades de materia y sus referencias (GSARE). Como lo sugieren sus nombres, son normas que definen los elementos constitutivos que conforman la estructura de los tres tipos de registro: de autoridad, de referencia y de referencia explicativa general. Las Reglas de Catalogación Angloamericanas. $2^{\text {a }}$ edición, revisión de 2002 y actualización de 2003 (AACR2), capítulo 23: Nombres geográficos y capítulo 24: Encabezamiento de entidades: se utilizó para la construcción de las autoridades, hacer las remisiones desde otras formas del nombre usadas y las referencias desde las formas variantes. Debe plantearse que estas reglas no contienen especificaciones ni ejemplos que indiquen y muestren cuál es el punto de acceso de reservas minerales, parques naturales, carreteras, túneles, bahías, ríos, etc.) que pueden ser tema de un documento y por ende, la biblioteca necesite ingresarlo como nombre geográfico. Por último, las Normas APA 6ta Edición: se utilizó esta norma para la citar y referenciar la bibliografía utilizada en la realización de la presente investigación y también para establecer el formato de las tablas y figuras.

\section{Lineamientos establecidos para normalizar los términos}

Reglas generales para la redacción de los descriptores

Se utilizarán los nombres propios como descriptores siempre y cuando el nombre forme parte significativa del contenido del documento y la práctica imponga su uso.

Forma del término: se utilizarán los sustantivos o formas sustantivadas. No incluir los llamados términos vacíos en indización (adjetivos, preposiciones, artículos, verbos en infinitivo y conjunciones).

Grafía del término: los descriptores se redactarán con letra inicial mayúscula respetando la grafía actual. Las variantes gráficas resultantes de la indización personalizada, por la carencia de un vocabulario controlado sobre nombres de topónimos, instituciones, yacimientos minerales, accidentes geográficos, obras ingenieriles, entre 
otros, estarán sujetos a control terminológico, durante el proceso de normalización en el que se redactará el término que las identificará inequívocamente.

- Número del término: se utilizará siempre el singular para el caso de los nombres propios que se utilicen como términos de indización.

- Forma de la entrada del término: Las entradas se harán en orden inverso para el caso de los nombres de descriptores físico naturales y algunos casos de obras ingenieriles. Los otros tipos de descriptores se harán en orden directo.

- Uso de signos de puntuación, siglas, abreviaturas y acrónimos: deben desarrollarse siempre que sea posible, de no ser así, se consignarán tal y como aparecen en el documento para garantizar su desarrollo posterior en el proceso de normalización. Se utilizarán paréntesis para aclarar los términos, en este caso para los descriptores físico naturales, obras ingenieriles, nombre de repartos, poblados, municipios, provincias, y países; y, los descriptores aplicados a las Ciencias Informáticas para esclarecer ubicaciones geográficas, nombre de software, lenguajes de programación, base de datos y aplicaciones informáticas.

- Se utilizarán como autoridades de materia a aquellos nombres propios que sean de uso corriente por los usuarios en las solicitudes de búsqueda.

\section{Ejemplos:}

En el área de la geología y minería: siempre se utilizarán los nombres de yacimientos, minas, canteras, formaciones geológicas, sectores, pozos, entre otros.

En el caso de la informática los nombres de los software y lenguajes de programación.

En el caso de la metalurgia los nombres de las plantas de las industrias en donde se desarrolla lo que se trata en el documento.

- Las entradas para onomásticos se harán en orden inverso: apellido, nombre; consignados además, como adiciones después de una coma: año de nacimiento y muerte si se conoce.

Ejemplo: Núñez Jiménez, Antonio, 1923-1998

\section{Reglas para la selección y formulación de descriptores institucionales}

Se entiende por entidad, a un organismo o grupo de personas conocidos por un nombre determinado que actúa o puede actuar con plena autonomía como una colectividad, considerada una unidad. Esta acepción abarca asociaciones, instituciones, firmas comerciales, empresas, gobiernos, entidades gubernamentales, proyectos, programas, instituciones religiosas, acontecimientos, así como competencias atléticas, exposiciones, expediciones, ferias y festivales, embarcaciones aéreas y marítimas. Las entradas institucionales se harán en orden directo.

Ejemplo: Hospital Pediátrico Pedro Sotto Alba

Se desarrollarán, siempre que sea posible las abreviaturas, siglas y acrónimos y la sigla se pondrá entre paréntesis con el lugar al cual pertenece la entidad.

Ejemplos: Empresa de Servicios del Níquel/no ESUNI

Empresa de Servicios del Níquel (ESUNI, Moa) 
Si en el documento apareciera la demarcación territorial de la entidad y no forma parte de su denominación, será consignada entre paréntesis para garantizar su identificación.

Ejemplo: Hospital General Gustavo Aldereguía Lima (Gibara)

\section{Reglas para la selección y formulación de descriptores geográficos}

Los descriptores geográficos, son los nombres propios de lugares o topónimos a los que se hace referencia en los documentos. Para su formulación se deben tener en cuenta las siguientes reglas:

Se seleccionarán los términos correspondientes a lugares tal y como aparecen en los documentos, consignando entre paréntesis el tipo de localidad y la jurisdicción a la que pertenecen acorde a la actual División Político Administrativa. En caso de no conocerse se recomienda utilizar el Nomenclátor de Nombres Geográficos Normalizados de Cuba editado por la Comisión Nacional de Nombres Geográficos (2013).

Ejemplo: Centeno (Consejo popular de Moa, Holguín)

Nombres de localidades, ciudades y municipios. Los nombres de localidades, ciudades y municipios se calificarán por la jurisdicción territorial mayor a la que pertenecen. Puntuación: encierre entre paréntesis las adiciones o calificadores a los nombres geográficos $\mathrm{AACR} 2,23.4 \mathrm{~A} 1$. Se escribirá con mayúscula solamente la primera letra de cada adición que no sea nombre jurisdiccional.

\section{Ejemplo: Manzanillo (Granma)}

Identificación de lugares. Para identificar un lugar, un barrio, una zona, etc., que se encuentra dentro de una ciudad, se antepondrá el nombre de la ciudad, al calificador de la jurisdicción provincial, separada con coma.

\section{Ejemplo: La Melba (Comunidad de Moa, Holguín)}

Aquellos lugares cuyos nombres tengan otros significados, se les pondrá un calificador seguido del nombre entre paréntesis.

$$
\text { Ejemplo: Calabazas (Consejo Popular de Sagua de Tánamo, Holguín) }
$$

En caso de coincidir en el descriptor, el nombre del municipio con el de la provincia se obviará ésta, como calificador. Es decir, aparecerá solo una vez la denominación respondiendo al municipio. Es aplicable también a la coincidencia de denominación entre municipio y provincia dentro de los calificadores.

$$
\begin{aligned}
& \text { Ejemplos: Holguín } \\
& \text { Bayamo (Granma) }
\end{aligned}
$$

Para el caso de los nombres geográficos de los países se elegirá el nombre de uso corriente y se realizará referencias de véase por el nombre oficial.

Ejemplos: Venezuela/ no República Bolivariana de Venezuela 
Para el caso de los descriptores físico naturales tales como valles, golfos, penínsulas, puntas, ríos, yacimientos, canteras, minas, sectores minerales, cuencas, lagos, bahías, se pospondrá el nombre genérico, es decir, el de la característica del accidente geográfico, separado con coma y espacio y conservando la mayúscula. Se reflejará en el calificador la jurisdicción actual a la que pertenecen.

\title{
Ejemplo: Botichino, Cantera (Jiguaní, Granma)
}

Para aquellos que abarcan más de un territorio se tendrán en cuenta las siguientes especificaciones:

Si está comprendido entre dos territorios (municipios, provincias o países) se consignarán en el calificador los nombres de esos territorios separados por un guion, colocando en primer término la jurisdicción donde el accidente ocupe mayor espacio, siempre y cuando estos no aparezcan reflejados en el nombre.

\section{Ejemplo: Macizo ofiolítico Moa-Baracoa \\ Piloto-Yarey, Campo mineral (Moa-Baracoa)}

Si abarca más de dos municipios de una misma provincia el calificador sólo incluirá el nombre de la provincia.

\section{Ejemplo: Yateras -Guaso, Trasvase (Guantánamo)}

Si abarca más de dos provincias, el calificador consignará el nombre de las provincias extremas.

\section{Ejemplo: Maestra, Sierra (Granma - Santiago de Cuba)}

Los descriptores geográficos correspondientes a obras de arquitectura e ingeniería como puentes, canales, presas, carreteras, túneles, parques eólicos, sitios patrimoniales, pozos, entre otros se redactarán en orden directo, tal y como aparecen en los documentos, y se encerrará entre paréntesis el lugar en que se encuentren, siempre que este no forme parte del nombre.

\section{Ejemplo: Carretera La Farola (Guantánamo)}

Nombres de lugares que incluyen o consisten en un término que indica accidente geográfico. Se registrarán en orden inverso, si el nombre de un lugar (nombre geográfico jurisdiccional) incluye o consiste en un término que indica accidente u otro objeto geográfico, transcríbalo tal como se encuentra en las fuentes de información.

$$
\text { Ejemplo: Alemán, Cerro (Venezuela) }
$$

\section{Reglas para la formulación de descriptores aplicados a la informática.}

Los descriptores correspondientes a nombres de aplicaciones, software, base de datos y lenguajes de programación, se redactarán utilizando el nombre del software en primer lugar y un calificador entre paréntesis con la designación a la que pertenece.

\author{
Ejemplo: Tierra (Software) \\ Java (Lenguaje de programación)
}


Reglas para la formulación de descriptores referentes a los nombres de las plantas industriales

Los descriptores correspondientes a los nombres de las plantas situadas en las industrias, se redactarán en orden directo, y el nombre de uso corriente, utilizando entre paréntesis un calificador para indicar en que fábrica se encuentra.

Ejemplo: Planta de espesadores (Fábrica Pedro Sotto Alba)

\section{Estructura del control de autoridades}

Para establecer el control de los puntos de acceso se siguió la siguiente estructura emitida por la IFLA y las GARE.

\section{Entrada de autoridad}

Encabezamiento autorizado

Está constituido por elementos como encabezamiento de autoridad y encabezamiento paralelo. Y por subelementos todos aquellos necesarios para identificar correctamente la autoridad.

\section{Nota de información}

Se utilizan cuando es necesario explicar las relaciones entre el encabezamiento aceptado y las citas de referencia. Están pensadas para ayudar al usuario y son de tipos:

- Notas de alcance para materias.

- Diferencias con otros términos.

- Organismos que cambian de nombre.

Área de cita de referencia de véase

Se consignan los encabezamientos no autorizados, introducidos por el signo < (equivale al usado por).

Variantes: Iniciales (acrónimos, formas más completas, formas más completas, formas menos completas, formas invertidas, ortografía).

Elementos: cita y referencia explicativa.

Área de cita de referencia de véase además

Se consignan encabezamientos también autorizados, introducidos por el signo $<<$ (véase además, T.G., T.E.)

Información: formas anteriores y posteriores del nombre, seudónimos usados como autorizados, cargos públicos (encabezamiento personal/corporativo).

Elementos: Cita y referencia explicativa.

Área de fuente (datos de control)

Elementos: Nombre del Centro Catalogador (símbolos), Reglas o normas de catalogación empleadas, Fecha de realización AAAAMMDD.

Área del número normalizado de datos de autoridad (ISADN). Intercambio y control internacional (sólo en caso de que exista).

Elemento: Número de control

\section{Entrada de referencia:}

Dirigen de un encabezamiento no aceptado a uno aceptado

De un encabezamiento aceptado a otros relacionados con él

Relaciones propuestas entre los términos:

$>$ Véase, Búsquese bajo

$<$ Usado por, Véase desde

>VÉase además

< Véase además desde precede al encabezamiento autorizado precede al encabezamiento variante precede al encabezamiento relacionado precede al encabezamiento autorizado 
Las GARR introdujeron símbolos para indicar las relaciones de "véase" y "véase además", consistentes en paréntesis angulares diferentes de los utilizados en la catalogación tradicional, pero admitidos por las normas de construcción de tesauros. En esta investigación para que resulte comprensible a los usuarios se usan los acrónimos utilizados por GSARE y que fueron adoptados como norma nacional en varios países de Europa según el estudio realizado por Martínez Tamayo y Mendes (2015), para remitir al punto de acceso autorizado e indicar las relaciones existentes entre los topónimos. En este caso las relaciones de "véase además", las relaciones existentes entre los términos y las notas de alcance no fueron utilizadas en esta investigación, por no contar con toda la información necesaria para establecer estas relaciones.

Tabla 2. Relaciones entre los términos

\begin{tabular}{|l|l|}
\hline Concepto & Acrónimo \\
\hline Equivalencia (sinonimia) & UP y USE \\
\hline
\end{tabular}

\section{Resultados de la investigación}

La propuesta de control de autoridades de nombres propios que constituyen materias está desarrollada para la carga de los metadatos de materia en el Repositorio Institucional Nínive de la Universidad de Moa Dr. Antonio Núñez Jiménez con el fin de proporcionar un instrumento útil de normalización de las autoridades de materia referida a los nombres propios de yacimientos, lugares, obras ingenieriles, accidentes geográficos, nombre de plantas industriales y nombre de software.

El control de autoridades compila un total de 389 términos, donde se presentan los términos autorizados y los no autorizados. Constituye una primera versión, en la edición de junio 2019.

La selección del nombre propio se basa en la condición de que solamente debe constituirse en autoridad una sola forma del nombre, independientemente de la cantidad de posibilidades bajo las cuales dicho nombre se conozca.

Para el desarrollo de la investigación se utilizó la literatura especializada en el tema abordado, y especialmente se tomó como referencia al Manual de Indización para Archivos del Archivo Nacional de Cuba, además de otras fuentes documentales, las cuales se adaptaron al contexto de la investigación.

La estructura del control de autoridades utilizada es la emitida por las GARE y revisada por la IFLA. El Control de Autoridades está constituido por dos partes: entrada de autoridad y entrada de referencia. Se organiza por tipo de descriptor y en orden alfabético. Para su uso será necesario tener en cuenta la siguiente simbología: relaciones de equivalencia: UP envía de los términos autorizados a los no autorizados, se escribe antecediendo al término no autorizado; y USE envía de los términos no autorizados a los autorizados, se escribe antecediendo al término autorizado.

El producto creado se encuentra en período de desarrollo y perfección, puesto que se decidió trabajar en una primera etapa con los nombres propios que son más utilizados por los usuarios de las especialidades de geología, minería, metalurgia e informática por lo que se irá actualizando a lo largo del tiempo, en la medida que vaya creciendo la colección documental y constituya el uso de estos términos una necesidad para la comunidad usuaria del repositorio. 
El catálogo se encuentra a disposición de los usuarios en el formato electrónico xml y se puede consultar al momento de completar el índice de materias del envío, también se encuentra en el formato de documento Word dirigido a los especialistas.

La actualización estará sujeta a revisiones sistemáticas por parte de las especialistas del Centro de Información Científico Técnica de la Universidad.

\section{Ejemplos de Entrada de Autoridad}

Tabla 3: Nombres de descriptores institucionales (plantas industriales)

\begin{tabular}{|l|l|}
\hline Áreas & Ejemplos \\
\hline 1. Encabezamiento de autoridad & ACINOX (Las Tunas) \\
\hline 2. Nota de información & $\begin{array}{l}\text { UP Empresa ACINOX } \\
\text { Empresa ACINOX LasTunas } \\
\text { Planta de acería }\end{array}$ \\
\hline $\begin{array}{l}\text { 3. Cita/s de referencia (usado por) } \\
\text { 4. Cita/s de referencia de relación } \\
\text { (véase además) }\end{array}$ & U. Moa; M.I 2002,20190520 \\
\hline $\begin{array}{l}\text { 5. Fuente } \\
\text { 6. ISADN }\end{array}$ & \\
\hline
\end{tabular}

Tabla 4. Nombres de descriptores geográficos físico naturales

\begin{tabular}{|l|l|}
\hline Áreas & Ejemplos \\
\hline 1. Encabezamiento de autoridad & $\begin{array}{l}\text { Parroquia Mene de Mauroa, } \\
\text { Campo mineral (Venezuela) }\end{array}$ \\
\hline 2. Nota de información & $\begin{array}{l}\text { UP Campo mineral Parroquia } \\
\text { Mene de Mauroa } \\
\text { Parroquia Mene de Mauroa }\end{array}$ \\
\hline 3. Cita/s de referencia (usado por) & U. Moa; M.I 2002,20190520 \\
\hline $\begin{array}{l}\text { 4. Cita/s de referencia de relación } \\
\text { (Véase además) }\end{array}$ & \\
\hline 5. Fuente & 6. ISADN \\
\hline
\end{tabular}

Ríos, cuencas, lagos, bahías, yacimientos, formaciones geológicas, campos minerales, canteras, minas, sectores y valles. 
Tabla 5: Nombres de descriptores geográficos referentes a obras ingenieriles

\begin{tabular}{|l|l|}
\hline Áreas & Ejemplos \\
\hline 1. Encabezamiento de autoridad & $\begin{array}{l}\text { Carretera La Farola } \\
\text { (Guantánamo) }\end{array}$ \\
\hline 2. Nota de información & \\
\hline $\begin{array}{l}\text { 3. Cita/s de referencia (usado por) } \\
\text { 4. Cita/s de referencia de relación } \\
\text { (Véase además) }\end{array}$ & UP La Farola \\
\hline 5. Fuente & U. Moa; M.I 2002,20190520 \\
\hline 6. ISADN & \\
\hline
\end{tabular}

Presas, túneles, carreteras, parques eólicos y pozos.

Tabla 6: Nombres de repartos, poblados, municipios, provincias, y países.

\begin{tabular}{|l|l|}
\hline Áreas & Ejemplos \\
\hline 1. Encabezamiento de autoridad & $\begin{array}{l}\text { Atlántico (Reparto de Moa, } \\
\text { Holguín) }\end{array}$ \\
\hline 2. Nota de información & UP Reparto Atlántico \\
\hline 3. Cita/s de referencia (usado por) & $\begin{array}{l}\text { 4. Cita/s de referencia de relación } \\
\text { (Véase además) }\end{array}$ \\
\hline 5. Fuente & U. Moa; M.I 2002,20190520 \\
\hline 6. ISADN & \\
\hline
\end{tabular}

Tabla 7: Nombres de descriptores aplicado a las Ciencias Informáticas

\begin{tabular}{|l|l|}
\hline Áreas & Ejemplos \\
\hline 1.Encabezamiento de autoridad & Java (Lenguaje de Programación) \\
\hline 2. Nota de información & \\
\hline $\begin{array}{l}\text { 3. Cita/s de referencia (usado por) } \\
\text { Java }\end{array}$ \\
\hline $\begin{array}{l}\text { 4. Cita/s de referencia de relación } \\
\text { (Véase además) }\end{array}$ & $\begin{array}{l}\text { UP Lenguaje de Programación } \\
\text { 5. Fuente }\end{array}$ \\
\hline 6. ISADN & U. Moa; M.l 2002,20190520 \\
\hline
\end{tabular}

Nombre de software, base de datos, aplicaciones informáticas y de lenguajes de programación. 


\section{Ejemplos de entrada de referencia}

Tabla 8: Nombres de descriptores institucionales (plantas industriales)

\begin{tabular}{|l|l|}
\hline Áreas & Ejemplos \\
\hline 1. Área de encabezamiento de referencia & Empresa ACINOX \\
\hline 2. Área de encabezamiento autorizado & USE ACINOX (Las Tunas) \\
\hline
\end{tabular}

\begin{tabular}{|l|l|}
\hline Áreas & Ejemplos \\
\hline 1. Área de encabezamiento de referencia & Empresa ACINOX Las Tunas \\
\hline 2. Área de encabezamiento autorizado & USE ACINOX (Las Tunas) \\
\hline
\end{tabular}

\begin{tabular}{|l|l|}
\hline Áreas & Ejemplos \\
\hline 1. Área de encabezamiento de referencia & Planta de acería \\
\hline 2. Área de encabezamiento autorizado & USE ACINOX (Las Tunas) \\
\hline
\end{tabular}

Tabla 9: Nombres de descriptores geográficos físico naturales

\begin{tabular}{|l|l|}
\hline Áreas & Ejemplos \\
\hline 1. Área de encabezamiento de referencia & $\begin{array}{l}\text { Campo mineral Parroquia } \\
\text { Mene de Mauroa }\end{array}$ \\
\hline 2. Área de encabezamiento autorizado & $\begin{array}{l}\text { USE Parroquia Mene de Mauroa, Campo } \\
\text { mineral (Venezuela) }\end{array}$ \\
\hline
\end{tabular}

\begin{tabular}{|l|l|}
\hline Áreas & Ejemplos \\
\hline 1. Área de encabezamiento de referencia & Parroquia Mene de Mauroa \\
\hline 2. Área de encabezamiento uniforme & $\begin{array}{c}\text { USE Parroquia Mene de Mauroa, Campo } \\
\text { mineral (Venezuela) }\end{array}$ \\
\hline
\end{tabular}

Ríos, cuencas, lagos, bahías, yacimientos, formaciones geológicas, campos minerales, canteras, minas, sectores y valles. 
Tabla 10 Nombre de descriptores geográficos referentes a obras ingenieriles

\begin{tabular}{|l|l|}
\hline Áreas & Ejemplos \\
\hline 1. Área de encabezamiento de referencia & La Farola \\
\hline 2. Área de encabezamiento autorizado & USE Carretera La Farola (Guantánamo) \\
\hline
\end{tabular}

Presas, túneles, carreteras, parques eólicos y pozos.

Tabla 11 Nombre de repartos, poblados, municipios, provincias y países

\begin{tabular}{|l|l|}
\hline Áreas & Ejemplos \\
\hline 1. Área de encabezamiento de referencia & Reparto Atlántico \\
\hline 2. Área de encabezamiento autorizado & USE Atlántico (Reparto de Moa, Holguín) \\
\hline
\end{tabular}

Tabla 12 Nombre de descriptores aplicados a las Ciencias Informáticas

\begin{tabular}{|l|l|}
\hline Áreas & Ejemplos \\
\hline $\begin{array}{l}\text { 1. Área de encabezamiento de } \\
\text { referencia }\end{array}$ & Lenguaje de Programación Java \\
\hline 2. Área de encabezamiento autorizado & $\begin{array}{l}\text { USE Java (Lenguaje de } \\
\text { Programación) }\end{array}$ \\
\hline
\end{tabular}

Nombre de software, bases de datos, y lenguajes de programación.

\section{Conclusiones}

Existen pocas investigaciones realizadas y lineamientos establecidos, a nivel nacional, para ejercer el control de autoridades de los nombres propios utilizados como encabezamientos de materia, lo que ocasionó para esta investigación un mayor estudio para concertar y tomar decisiones.

La compilación de los términos realizada arrojó un alto nivel de inconsistencia presente en los índices de materia, donde se revela que las causas están dadas por: el uso indistinto de nombres propios por parte de los autores de los documentos y de los indizadores al utilizar estos términos como encabezamientos de materia, en el proceso de indización; la falta de lineamientos y políticas precisas para realizar la indización de los documentos utilizando este tipo de términos y la inexistencia de un catálogo de autoridades de nombres propios.

La investigación permitió normalizar 389 nombres propios a partir de 25 lineamientos establecidos y elaborar un catálogo de autoridades que si bien no es una obra concluida, aporta al desarrollo del Repositorio Institucional Nínive de la Universidad de Moa Dr. Antonio Núñez Jiménez favoreciendo la búsqueda y recuperación de la información. 


\section{Q Referencias Bibliográficas}

》Acevedo Rodríguez, Pedro y Julia de León Herrero. 2013. Los nombres geográficos de Cuba. La Habana: Pueblo y Educación.

» Bacallao González, Wilfredo; Xiomara Garzón Montes de Oca y Ronald BacaIlao González. 2011. Diccionario Geográfico de Holguín. Holguín: PNUD.

»Barrionuevo Almuzara, Leticia, María Luisa Alvite Díez y Blanca Rodríguez Bravo. 2011. Análisis del control de autoridades en los repositorios universitarios españoles. En Congreso ISKO 20 años del Capítulo Español de Isko (10ㅇ: 30 junio al 1 julio de 2011: El Ferrol, La Coruña). Trabajos presentados. Ferrol: Universidade da Coruña, Facultad de Humanidades-Campus de Ferrol. <http://buleria. unileon.es/handle/10612/2893> [Consulta: 29 abril 2020].

» Biblioteca Nacional de España. 2013. Manual de indización de Encabezamientos de materia. España. <http://www.bne.es/es/Micrositios/Guias/ManualMaterias/Docs/EMBNE.pdf> [Consulta: 29 abril 2020].

"Comisión Nacional de Nombres Geográficos de Cuba. 2013. Los Nombres Geográficos de Cuba. La Habana: Pueblo y Educación.

"Comisión Provincial de Nombres Geográficos. 2013. Nomenclator de Nombres Geográficos Normalizados de Holguín. Holguín: Oficina Territorial de Cartografía y Geodesia.

"Díaz Rodríguez, Yoselyn. 2012. Control de autoridades de nombres personales de autores cubanos en ciencias de la salud. En ACIMED. Vol. 23, no 1, 35-44.<http:// scielo.sld.cu/scielo.php?script=sci_arttext\&pid=S1024-94352012000100004\&l ng=es\&tlng=es $>$ [Consulta: 29 abril 2020].

»Duperet Cabrera, Elaine; Denis Gabriel Pérez Martínez; Mirtha Yris Cedeño Rodríguez; Adrián Ramírez Mustelier y Luis Alberto Montoya Acosta. 2015. Importancia de los repositorios para preservar y recuperar la información. En Medisan. Vol. 19, no. 10, 3080-3087. <http://scielo.sld.cu/scielo.php?pid=S102930192015001000014\&script=sci_arttext\&tlng=en> [Consulta: 29 abril 2020].

» Firpo, Susana. 2014. Manual de procedimientos para el control de autoridades. Nombres geográficos. Buenos Aires: Biblioteca Nacional de la República Argentina. Dirección Técnica Bibliotecológica. División Control de Autoridades. <https://escritoriopt.bn.gov.ar/pdfs/BNMM-DCA-Manual-NG-v2-201410.pdf> [Consulta: 29 abril 2020].

"García Rodríguez, Suledny. 2016. Propuesta de normalización para el control de autoridades en la Red TIC Cuba. Santa Clara: Universidad Central "Marta Abreu" de Las Villas, 2016. 115 p. Tesis de Pregrado-Licenciatura.<http://dspace.uclv.edu.cu/bitstream/handle/123456789/6618/ Garc\%c3\%ada\%2oRodr\%c3\%adguez\%2c\%20Suledny\%20\%2806_16\%29. pdf? sequence $=1$ \&isAllowed $=y>$ [Consulta: 29 abril 2020].

"Maldonado Martínez, Ángeles y Luis Rodríguez Yunta. 2004. Tesauro ISOC de Topónimos 2004 (Edición abreviada). Madrid: Centro de Información y Documentación Científica CINDOC. CSIC. < http://digital.csic.es/bitstream/10261/30253/1/TesauroToponimos2004.pdf > [Consulta: 29 abril 2020]. 
» Martínez Saldaña, Julia Margarita. 2006. Control de autoridades. Metodología para crear registros de autoridad catalográfica. San Luis Potosí: Tecnológico de Monterrey, 2006. 112 p. Tesis de Maestría. <https://repositorio.tec.mx/bitstream/handle/11285/567948/DocsTec_5497.pdf? sequence $=1 \&$ isAllowed $=y>$ [Consulta: 29 abril 2020].

» Martínez Tamayo, Ana María; Norma E. Mangiaterra; Cristina A. Ristuccia; Mariana Pichinini y Mónica G Pené. 1997. Control de autoridades en catálogos en línea. En Investigación bibliotecológica. Vol. 11, no. 23, 80-101. <http://rev-ib. unam.mx/ib/index.php/ib/article/view/3848/3401 > [Consulta: 29 abril 2020].

" Martínez Tamayo, Ana María y Paola Verónica Mendes. 2015. Diseño y desarrollo de tesauros. La Plata: Universidad Nacional de La Plata. Facultad de Humanidades y Ciencias de la Educación. <http://www.memoria.fahce.unlp.edu.ar/ libros/pm.273/pm.273.pdf > [Consulta: 29 abril 2020].

»Mesa León, Marisol; Elvira Corbelle Sanjurjo; Alba Gilda Dreke Alfonso; Miriam Ruíz Meriño y Jorge Macle Cruz. 2007. Manual de indización para archivos. La Habana: Archivo Nacional de la República de Cuba. <https://www.ica.org/ sites/default/files/ALA\%20Manual\%20de\%2olndizacion\%2opara\%2oarchivos.pdf> [Consulta: 29 abril 2020].

"Núñez Amaro, Sonia. 2006. El control de autoridades: definiciones y apuntes históricos. En Bibliotecas. Anales de Investigación. No. 2, 42-53. <https:// www.researchgate.net/profile/Sonia_Nunez_Amaro/publication/281554624_ El_control_de_autoridades_definiciones_y_apuntes_historicos/ links/55ed84aco8ae21do99c75bef/El-control-de-autoridades-definiciones-yapuntes-historicos.pdf> [Consulta: 29 abril 2020].

»Núñez Amaro, Sonia. 2007. El Control de Autoridades en la Biblioteca Nacional José Martí y en el Sistema Nacional de Bibliotecas Públicas: propuesta de implementación. En Bibliotecas. Anales de Investigación. No.3, 41-75. < https://dialnet.unirioja.es/servlet/articulo?codigo $=6099043>$ [Consulta: 29 abril 2020].

»Pugni, Nora Susana. 2011. Proceso de creación de una lista de autoridades de Nombres geográficos de Argentina. La Plata: Universidad Nacional de La Plata. Facultad de Humanidades y Ciencias de la Educación, 2011. 85 p. Tesis de Licenciatura. <http://www.memoria.fahce.unlp.edu.ar/ library? $\mathrm{a}=\mathrm{d} \& \mathrm{c}=$ tesis \&d=Jte801 > [Consulta: 29 abril 2020]. 
